\title{
COUPLED ELECTROMECHANICAL NUMERICAL MODELLING OF PiezoeleCtric Vibration ENERgy HaRVeSTERS
}

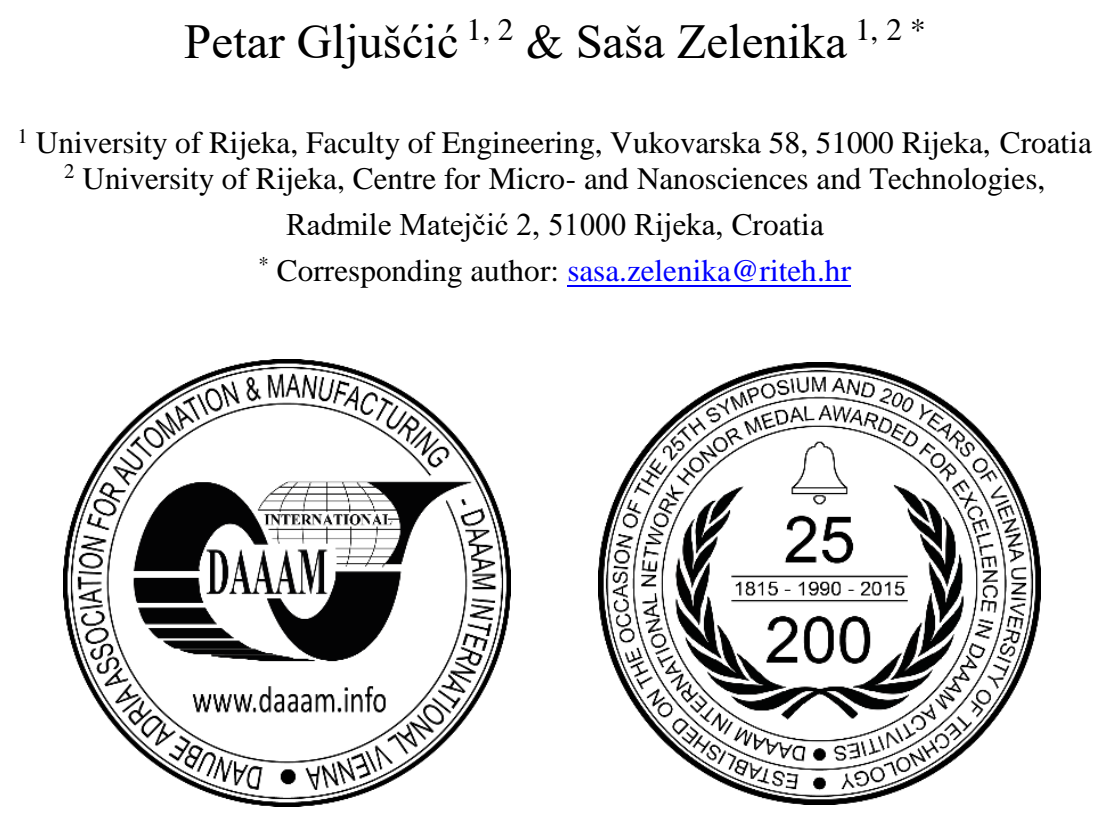

This Publication has to be referred as: Gljuscic, P[etar] \& Zelenika, S[asa] (2018). Coupled Electromechanical Numerical Modelling of Piezoelectric Vibration Energy Harvesters, Proceedings of the 29th DAAAM International Symposium, pp.0009-0015, B. Katalinic (Ed.), Published by DAAAM International, ISBN 978-3-902734-20-4, ISSN 1726-9679, Vienna, Austria

DOI: $10.2507 / 29$ th.daaam.proceedings.002

\begin{abstract}
Energy harvesting is the process of collecting low-level ambient energy and converting it into electrical energy to be used for powering miniaturized autonomous devices, sensor networks, wearable electronics or Internet-of-Things components. The use of the pervasive kinetic energy, converted into electrical energy, is of special interest in this frame. The possibility to use bimorph piezoelectric cantilevers to convert ambient vibrations to electrical energy is therefore thoroughly analyzed in this work. A reliable modelling tool for optimizing the design of the miniature harvesters to be used in a broad frequency range, while maximizing the obtained powers, is hence needed. The problem complexity is induced by the necessity to simulate the dynamic response of the considered harvesting devices via a coupled electromechanical model. The recently developed comprehensive coupled analytical model based on distributed parameters is thus used as a benchmark to verify and tune suitable finite element (FE) numerical models. Modal (allowing to determine the mechanical dynamic response and the respective eigenfrequencies), harmonic (resulting in coupled frequency response functions) as well as linear and nonlinear transient FE analyses (resulting in dynamic responses under forced excitation at discrete time steps, including geometric nonlinearities) are therefore performed and complex dynamics effects are observed.
\end{abstract}

Keywords: energy harvesting; piezoelectric bimorph cantilevers; coupled analysis; design; frequency bandwidth; wearable technology

\section{Introduction}

Energy harvesting is the process of collecting low-level ambient energy and converting it into electrical energy to be used for powering miniaturized autonomous devices, sensor networks, wearable electronics or Internet-of-Things components. 
The energy sources generally considered for this purpose comprise solar/light energy, waste heat, kinetic energy and radio-frequency [1], [2]. Approaches to the collection of such energy largely depend on the energy form, e.g. photovoltaic modules are used for harvesting solar energy, whereas thermoelectric generators are used when thermal energy sources are considered.

Kinetic energy, ubiquitous in the environment and usually caused by the motion of living beings or machinery, is of special interest in this framework. In fact, kinetic energy can easily be converted into electrical energy by using piezoelectric transducers, which proved to be advantageous due to design simplicity, miniaturization and integration potential and high energy density. The main task in designing such devices is, in turn, the achievement of maximum efficiency for the given working conditions and the available space [1], [2].

The possibility to use bimorph piezoelectric cantilevers to convert ambient vibrations to electrical energy is hence thoroughly analyzed in this work. The studied class of devices consists of two layers of piezoelectric material on a metallic substrate, it is fixed on one end while a tip mass, placed on its free end, is used to magnify the deflections and tune the eigenfrequency of the device to that of the base excitation (Figure 1). When the cantilever is dynamically excited, the mechanical energy resulting from the deformation of the piezoelectric layers is therefore converted via the electromechanical coupling effect into electrical energy, thus generating a voltage difference between the electrodes on the surfaces of the piezoelectric layers [1], [2].

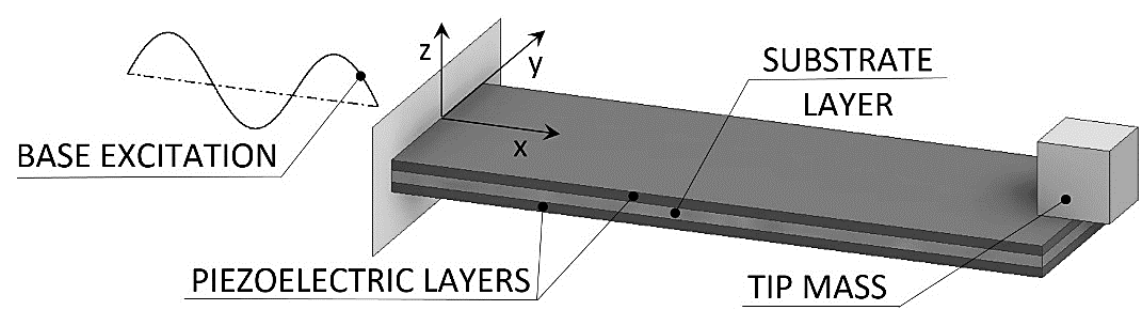

Fig. 1. Piezoelectric bimorph

One of the major drawbacks of piezoelectric bimorphs used as energy harvesting devices is the narrow area of optimal operation around the eigenfrequencies of the specific device. The voltage level achieved within this narrow area is high, but it rapidly decreases with even a slight variation of the excitation frequency. There are some vibration sources, such as e.g. rotating machinery, suitable for such type of operation, but the predominant vibration sources are characterized by an excitation that varies in frequency and in intensity, which drastically decreased the energy conversion efficiency of the energy harvesting devices [1], [2].

The widening of the optimal operations area of the piezoelectric harvesters can be approached in several ways, where the most promising ones are:

- changing the conditions around the cantilever free end (e.g. via active tuning or damping control), or

- changing the cantilever's geometry (by using complex geometries with bi-stable or nonlinear responses, or by resorting to a large number of differently tuned cantilevers) [1], [2].

To be able to model the response of the resulting different harvesters' configuration so as to maximize the obtainable voltage and power levels, a suitable modelling tool is hence needed. Although several modelling approaches are suggested in literature [1], [2], the most comprehensive one, comprising the coupled electromechanical response of piezoelectric energy harvesters, is the recently developed "coupled modal electromechanical distributed parameter model" (CMEDM) [3]. In fact, with respect to previous lumped parameters models, CMEDM is based on distributed parameters and on solving canonically the dynamics of the Euler-Bernoulli beam [4], while concurrently including the piezoelectric backward coupling effect, the influence of the tip mass as well as the damping effects of the system itself and of the air surrounding it. The resulting output voltage amplitude $\alpha_{s}$ of the piezoelectric energy harvester can be described as [3]:

$$
\alpha_{s}(\omega)=\frac{\sum_{r=1}^{\infty} \frac{j \omega \kappa_{r} \sigma_{r}}{\omega_{r}^{2}-\omega^{2}+j 2 \zeta_{r} \omega_{r} \omega}}{\frac{1}{R_{l}}+j \omega \frac{C_{\tilde{p}}}{2}+\sum_{r=1}^{\infty} \frac{j \omega \kappa_{r} \chi_{r}^{S}}{\omega_{r}^{2}-\omega^{2}+j 2 \zeta_{r} \omega_{r} \omega}} e^{j \omega t}
$$

where $j$ is the imaginary unit, $\omega\left(\mathrm{s}^{-1}\right)$ is the excitation frequency close to harvester's eigenfrequency $\omega_{r}\left(\mathrm{~s}^{-1}\right), \kappa_{r}$ is the forward coupling term, $\sigma_{r}$ is the translational component of the excitation, $\zeta_{r}$ is mechanical damping, $R_{1}(\Omega)$ is the external electrical load acting on the system, $C_{\tilde{p}},(\mathrm{~F})$ is the capacitance of the piezoelectric material and $\chi_{r}^{s}$ is the modal coupling term [3]. In literature it was experimentally shown that, in terms of achieved peak voltages at a determined eigenfrequency, CMEDM provides reliable results for bimorph cantilevers with a constant rectangular cross-section [3], [5]. Since, however, the effects of the mentioned geometry and other harvester parameters' variations on the electromechanical response of the device cannot be analyzed by using (1), a more elaborated tool based on a comprehensive numerical analysis of the response of the piezoelectric harvesters has to be used. 
A finite element model (FEM) is hence developed and tuned with the proven CMEDM, enabling therefore a more efficient approach to the development of devices with an optimal response in real operating conditions.

\section{Numerical Model}

The main aim of using FEM analysis is to allow a straightforward analysis of the impact of the variation of design parameters on the response of piezoelectric harvesters, thus overcoming the limitations of CMEDM. To analyze numerically the complex electromechanical coupling of the considered piezoelectric bimorph cantilevers, several analyses have, however, to be carried out, i.e.:

- the modal analysis,

- the coupled harmonic analysis and

- the coupled transient analysis

have to be performed.

The numerical model is developed by using the ANSYS ${ }^{\circledR}$ software and specifically its parametric design language (APDL) [5], [6], where a basic multivolume block model of the device is generated and all the material parameters are defined. Element types used to model the considered harvester include (cf. ANSYS ${ }^{\circledR}$ software help module):

- SOLID226 - a standard ANSYS $^{\circledR}$ prismatic element able to simulate piezoelectric, piezoresistive and thermoelectric material properties,

- SOLID186 standard prismatic element, used to model the substrate and the tip mass,

- CIRCU94 element used in the harmonic and the transient analyses for the simulation of the electrical (load) resistance.

The implemented boundary conditions are equivalent to those used in the CMEDM model, i.e., the fixed end of the cantilever is clamped at the substrate layer, while the other end is free. It is to be noted here that clamping the cantilever at the piezoelectric layers influences significantly the results, shifting the peak response towards higher frequencies with respect to that of the CMEDM model. What is more, it is established that the obtained model (Figure 2) is relatively insensitive to element meshing size, with negligible discrepancies in all the considered cases.

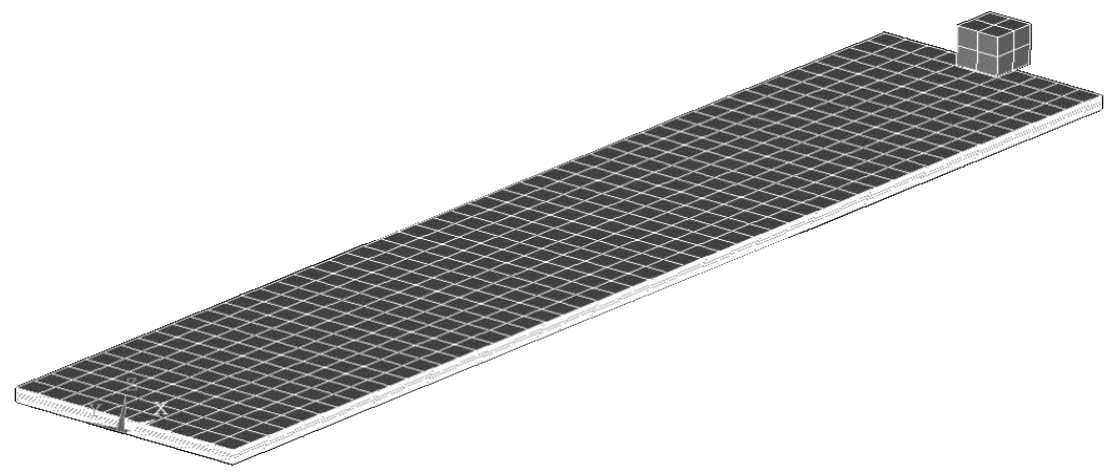

Fig. 2. Model of piezoelectric bimorph generated in ANSYS ${ }^{\circledR}$

As described in more detail in the following sections, two cases of cantilever's boundary conditions are hence considered: one with and the other one without the tip mass.

\subsection{Modal and coupled harmonic analyses}

The main purpose of the modal analysis is the determination of the mechanical dynamics response and the respective eigenfrequencies and modal shapes of the considered piezoelectric bimorph. The results of the modal analysis are then used not only as a preliminary prove of the validity of the numerical model as compared with CMEDM, but also as a framework for the subsequent frequency sweep in the harmonic analysis. In this frame, the work is based mainly on considering the first modal shape, since the first eigenfrequency is generally used in practice allowing to attain the highest deformations and therefore the biggest achievable output voltages.

In a first instance, a pure mechanical response of the bimorph, setting to zero the piezoelectricity coefficient $e_{31}$ in the material properties of the piezoelectric layers, eliminating in this way the effects of electromechanical coupling, is calculated. The sparse direct matrix solver is defined in this frame according to ANSYS ${ }^{\circledR}$ recommendation, since it is the most robust solver type available in $\mathrm{ANSYS}^{\circledR}$ and is based on a direct elimination of equations, as opposed to solvers where a more cumbersome iterative process is used [3], [6].

The thus attained mechanical eigenfrequencies for the two considered cases (cantilever of dimensions length $\mathrm{x}$ width $\mathrm{x}$ thickness $=100 \times 20 \times 1.3 \mathrm{~mm}$ with and without tip mass), are reported in Table 1. Is can be observed that the FEM results match excellently the CMEDM values, proving thus the validity of the developed numerical model. 


\begin{tabular}{|c|c|c|}
\hline & $\begin{array}{c}\text { CMEDM mechanical } \\
\text { eigenfrequency, } \boldsymbol{f}_{\text {mech }}(\mathbf{H z})\end{array}$ & $\begin{array}{c}\text { FEM mechanical } \\
\text { eigenfrequency, } \boldsymbol{f}_{\text {mech }}(\mathbf{H z})\end{array}$ \\
\hline Cantilever without tip mass & $62.49 \mathrm{~Hz}$ & $62.49 \mathrm{~Hz}$ \\
\hline Cantilever with tip mass $(1 \mathrm{~g})$ & $57.10 \mathrm{~Hz}$ & $57.17 \mathrm{~Hz}$ \\
\hline
\end{tabular}

Table 1. Mechanical eigenfrequencies comparison

To determine the coupled electromechanical response of the bimorph cantilever, i.e., the respective coupled frequency response functions (FRFs), a coupled harmonic analysis is performed next. A frequency spectrum around the first eigenfrequency of the harvester, as derived from the modal analysis, is hence considered, and thus a frequency sweep from 45 to $80 \mathrm{~Hz}$ is used. In this phase, a load resistance is also introduced into the model by using the CIRCU94 elements between the two nodes where the output voltage is measured. To match the load resistance used in the CMEDM model, the resistance is set to $R_{1}=100 \mathrm{k} \Omega$. It is to be noted that, while performing the harmonic analyses, the nodes on the surfaces of the piezoelectric layers are no longer separated. In fact, in order to simulate the electrodes on the piezoelectric layers, the nodes on each of the four surfaces have to be connected to the load resistance element (CIRCU94) nodes by using the respective VOLT degrees of freedom. The outer electrodes are connected, accordingly, to one resistor node, while the electrodes on the surfaces of the piezoelectric material towards the metallic substrate layer are connected to the other node, thus simulating the serial connection of the electrodes. Before initializing the analysis, the voltage value on one of the load resistance node has to be set to $0 \mathrm{~V}$ [5]. To match the condition used in the CMEDM model, a harmonic excitation is then defined in the form of an acceleration of cantilever's base of $1 \mathrm{~m} / \mathrm{s}^{2}$ in the $z$ direction (cf. Figure 1). The other boundary conditions at the fixed end of the cantilever coincide with those used in the modal analysis [3], [5], [6].

The harmonic analysis requires the definition of the damping, which is a very complex phenomenon in distributed mechanical systems also because the energy dissipation problem in materials is currently not entirely explored. One possible approach to the approximation of damping in such systems is the usage of the so-called Rayleigh damping, commonly used in FEM analyses. Rayleigh damping involves the calculation of the damping matrix $\mathbf{B}_{\mathrm{d}}$ as a sum of the mass $\mathbf{M}$ and stiffness $\mathbf{K}_{\mathrm{S}}$ matrices, multiplied by the corresponding damping constants $\alpha$ and $\beta$ [7]:

$$
\mathbf{B}_{\mathbf{d}}=\alpha \mathbf{M}+\beta \mathbf{K}_{\mathrm{s}}
$$

where, by using the experimentally determined damping coefficient $\zeta$ as well as the first two eigenfrequencies $f_{1}$ and $f_{2}$ obtained via the modal analysis, the damping constants can be calculated from:

$$
\begin{aligned}
& \frac{\alpha}{4 \pi f_{1}}+\beta \pi f_{1}=\zeta \\
& \frac{\alpha}{4 \pi f_{2}}+\beta \pi f_{2}=\zeta
\end{aligned}
$$

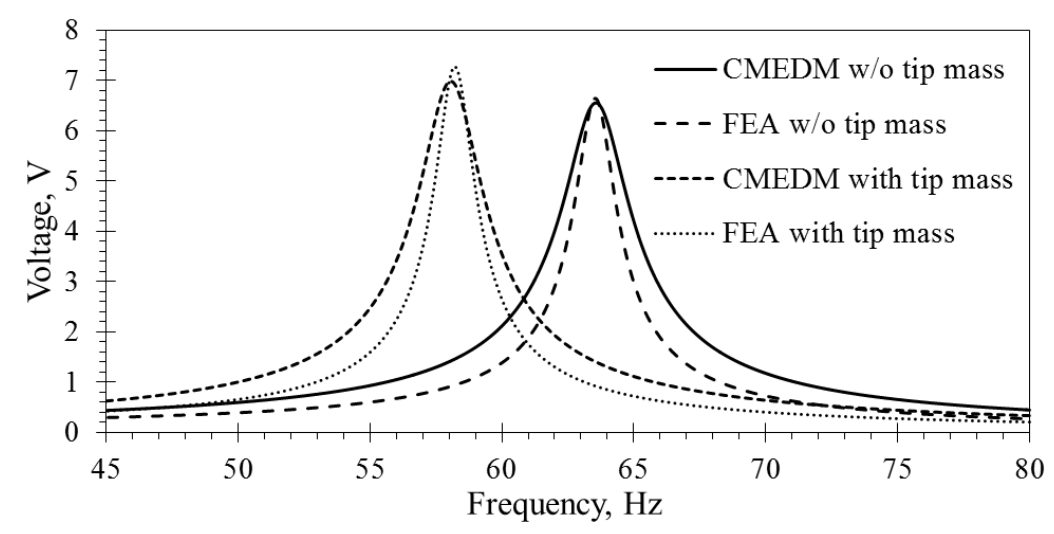

Fig. 3. Coupled electromechanical response comparison between CMEDM and FEM

The results of the coupled harmonic analyses, i.e. the electromechanical FEM FRFs attained for both considered bimorph cantilever cases are shown in Figure 3, where they are compared with the corresponding responses derived by employing the CMEDM model. It can therefore be inferred that a close match of CMEDM and FEM results is obtained in terms of the eigenfrequencies (Table 2) and maximal voltage levels, albeit a clear narrowing of the overall FEM response curve, to be further investigated with experimentally obtained data, is also seen. 
In this frame it has to be noted that, the further away from the eigenfrequency, less relevant the data are for the energy harvesting application, since in the herein considered cases the most important parameters are the peak voltage values at a certain well determined eigenfrequency. On the other hand, as expected, when a tip mass is added, an increase in output voltages with a concurrent decrease of the eigenfrequencies is achieved. It is especially important to note also that, due to the electromechanical coupling effects, i.e., the influence of electrical parameters on the response of the considered system, a significant (up to $1.1 \mathrm{~Hz}$ ) difference in the eigenfrequencies with respect to those obtained via a purely mechanical response (i.e. the modal analyses), is generated (cf. the results reported in Table 2 in comparison to those of Table 1).

\begin{tabular}{|c|c|c|}
\hline & $\begin{array}{c}\text { CMEDM coupled } \\
\text { eigenfrequency, } \boldsymbol{f}_{\text {coupl }}(\mathbf{H z})\end{array}$ & $\begin{array}{c}\text { FEM coupled } \\
\text { eigenfrequency, } \boldsymbol{f}_{\text {coupl }}(\mathbf{H z})\end{array}$ \\
\hline Cantilever without tip mass & $63.54 \mathrm{~Hz}$ & $63.6 \mathrm{~Hz}$ \\
\hline Cantilever with tip mass $(1 \mathrm{~g})$ & $58.06 \mathrm{~Hz}$ & $58.3 \mathrm{~Hz}$ \\
\hline
\end{tabular}

Table 2. Coupled eigenfrequency differences between CMEDM and FEM models

\subsection{Transient analyses and geometrical nonlinearities}

The transient analysis is used to model the dynamics responses of the harvester under forced excitation in precisely defined discrete time increments and it hence generates steady state results for every time iteration [5]. Since, however, in this case it is impossible to simply set the excitation amplitude as in the harmonic analysis, the transient analysis implies the need to define a sinusoidal excitation profile, generated in MATLAB ${ }^{\circledR}$, which is then imported in ANSYS $^{\circledR}$ for every time-step of the analysis. For this purpose, the excitation profile is imported in ANSYS ${ }^{\circledR}$ in tabular form and implemented in each time-step via a *DO loop. The loop introduces new values of the translation of cantilever's base, initially set to zero, for each time increment according to the excitation profile, and rewrites them over the previously set values.

It is important to note here that, due to the amount of time needed for the execution of each analysis, the transient analyses are performed within a narrow range of frequencies around the first eigenfrequency; for the same reason, this type of analyses are performed in a first instance only for the case of the cantilever without the tip mass. What is more, to ensure a correct execution of the analyses, i.e. the achievement of the steady state conditions, a sufficient number of cycles at each frequency is needed. It is therefore determined that the number of points defining one period of the sinusoidal excitation should be at least $N=16$, the frequency increment is to be limited to $\Delta f=0.05 \mathrm{~Hz}$, while the number of excitation cycles at each frequency has to be at least $n=16$. The mentioned damping coefficients ALPHAD and BETAD are then set to the same values as in the harmonic analyses (cf. (2) - (4)), while the $2^{\text {nd }}$ order transient integration parameters ALPHA and DELTA, as well as the $1^{\text {st }}$ order transient integration parameter THETA, are set, according to the ANSYS $^{\circledR}$ recommendations for the piezoelectric analyses, as $0.25,0.5$ and 0.5 respectively. The model of the cantilever itself, the load resistance settings, as well as the electrodes' couplings are, in turn, the same as in the harmonic analyses. After every base excitation step, the obtained results are finally saved along with the respective time interval setting.

Especially important in this whole framework is also the fact that in several types of structures, e.g. shells or, as in the herein considered case, beams, i.e., cantilevers, the occurrence of large deflections (larger than 5\% of cantilever's length) causes the cross sections of the analyzed structure to rotate with respect to each other. The resulting responses are therefore no longer predictable by the assumptions of the linearized Euler-Bernoulli theory. In ANSYS ${ }^{\circledR}$ the NLGEOM option allows the inclusion of the effects of such geometrical nonlinearities in the transient analyses. In this work, linear and a nonlinear transient analyses are thus performed, and the obtained results are compared between them, as well as against the results attained via the coupled harmonic and the CMEDM analyses.

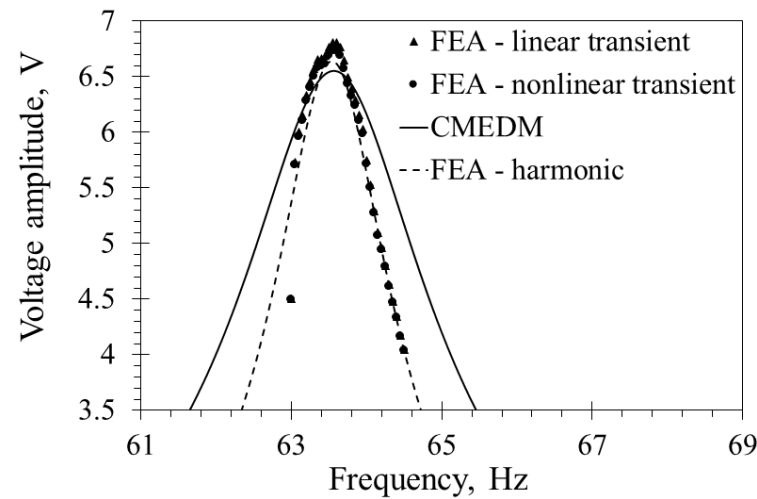

(a)

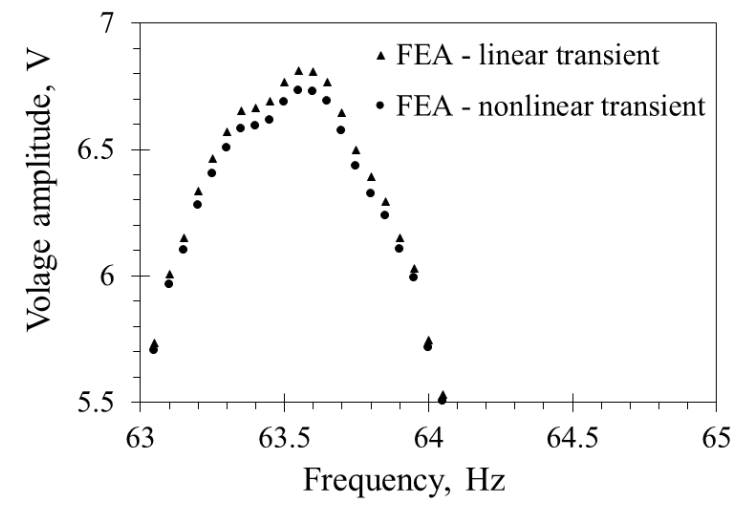

(b)

Fig. 4. Comparison of transient with harmonic and CMEDM responses

(a) and of linear and nonlinear transient results (b) 
The linear and nonlinear transient analyses results are obtained as time related voltage values, which, to be compared with the harmonic responses, need to be transformed from the time domain to a FRF representation. The finally attained results are shown in Figure 4. With respect to the results achieved via the previously performed analyses, and within the limits evidenced in the discussion related to Figure 3, a good match of the coupled eigenfrequency resulting from both transient analyses types can hence be observed in Figure 4a. In terms of the output voltages, however, an increase in the transient analyses vales (ca. 0.1 to $0.2 \mathrm{~V}$ with respect to the CMEDM results) can be observed.

The physical background of this difference is not yet clear and, together with a slight "glitch" observable in the region approaching the maximal voltages, will again be examined via a comparison with experimental results. When a more detailed comparison of the results of the nonlinear vs. the linear transient analyses is performed (Figure 4b), it can be observed that the nonlinear analysis results in slightly lower maximal voltage levels, whereas both the eigenfrequency value and the overall system responses are, basically, coinciding.

\section{Future work}

A possible practical application of piezoelectric bimorph energy harvesters that we are currently actively considering in collaboration with medical institutions, is that of wearable devices. In fact, in this case devices and sensors, able to acquire, analyze and transmit data, could be worn as clothing or as a kind of accessories such as e.g. watches. Wearable devices are especially interesting in the field of telemedicine, where they could be used for real-time remote monitoring of patients, including an early warning system in case of an aggravation of patients' physiological states. Wearable sensors could in this frame be used to monitor e.g. blood pressure, pulse or body temperature, accelerometers could be used as fall detectors [8], while microneedles could even allow autonomous and automated drug delivery [9].

An initial study is hence performed to analyze the possibilities of implementing piezoelectric bimorph energy harvesters in a watch-like device intended to be used as a power supply for medical wearable technology devices, resulting in prototype designs shown in Figure 5 [10]. The conceived designs would allow the conversion of kinetic energy generated by the human arm movement into electrical energy, which could then be used to power wearable sensors. Future work in this frame includes further theoretical analyses as well as the practical development and optimization of these or other similar devices as wearable energy sources that could enable the creation of autonomous wearable medical devices.
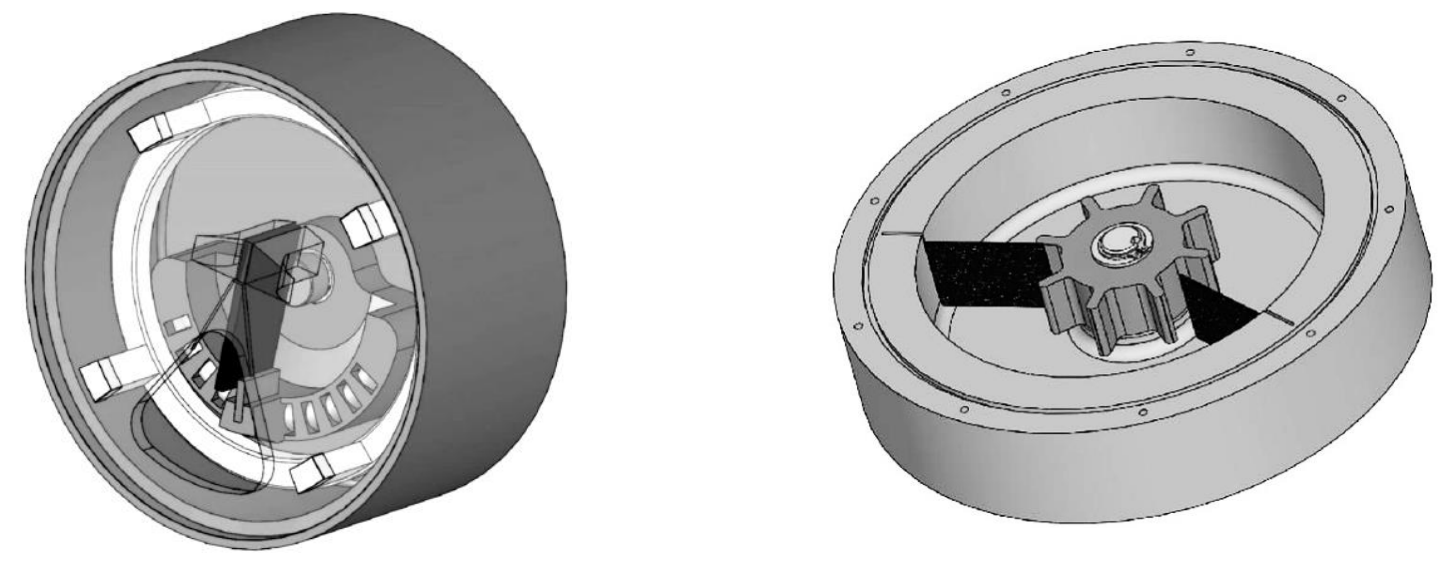

Fig. 5. Possible designs of piezoelectric energy harvesters in watch-like wearable devices [10]

\section{Conclusions}

The aim of this work is the development of reliable tools for the analysis and the optimization of the design and performances of piezoelectric energy harvesting devices. A comparison between the established CMEDM model of the dynamics response of bimorph piezoelectric cantilevers and advanced numerical (FEM) modelling is hence performed. A numerical model is thus created in ANSYS ${ }^{\circledR}$, and modal, coupled harmonic, as well as linear and nonlinear transient analyses are carried out, enabling a thorough study of the complex electromechanical coupling behavior of the studied class of devices. It is established that the FEM results closely match those obtained via CMEDM, creating the preconditions for an easier and faster consideration of different geometries as well as of the variation of other design parameters of the piezoelectric harvesters.

The study needs, however, to be complemented also with a detailed experimental validation so as to clarify the inconsistencies between some of the obtained results in terms of slight discrepancies in the obtained peak voltages and bigger differences in the width of the response curves. All this will allow optimizing and dimensionally miniaturizing the design of piezoelectric energy harvesters while broadening their usable bandwidths and maximizing the obtained powers, thus eventually allowing to use such optimized design configurations in integrated autonomous wearable medical devices. 


\section{Acknowledgments}

The work described in this paper is enabled by using the equipment funded via the ERDF project RC.2.2.06-0001 "Research Infrastructure for Campus-based Laboratories at the University of Rijeka - RISK" as well as via the support of the University of Rijeka grant 13.09.1.2.09 "Characterisation and Modelling of Materials and Devices for Innovative Applications".

\section{References}

[1] Kaźmierski, T. J. \& Beeby, S. (Ed.) (2011). Energy harvesting systems principles, modeling and applications, Springer, ISBN: 978-1-4419-7565-2, New York, USA

[2] Prya, S. \& Inman, D. (Ed.) (2009). Energy harvesting technologies, Springer, ISBN: 978-0-387-76463-4, New York, USA

[3] Erturk, A. \& Inman, D. J. (2009). An experimentally validated bimorph cantilever model for piezoelectric energy harvesting from base excitations, Smart Mater \& Struct, Vol. 18, No. 2, 025009 (18pp), ISSN: 1361-665X

[4] Meirovitch, L. (2001). Fundamentals of vibrations, McGraw Hill, ISBN: 0-07-118174-1, Boston, USA

[5] Blažević, D. (2014). Analysis of mechanical aspects in the design of vibration energy harvesters, D. Sc. Dissertation, Faculty of Engineering, University of Rijeka, Rijeka, Croatia

[6] Benasciutti, D., Moro, L., Zelenika, S. \& Brusa, E. (2010). Vibration energy scavenging via piezoelectric bimorphs of optimized shapes, Microsyst Technol, Vol. 16, No. 5, pp. 657-668, ISSN: 1432-1858

[7] Takács, G. \& Rohal'-Ilkiv, B. (2012). Model predictive vibration control, Springer-Verlag, ISBN: 978-1-44712332-3, London, UK

[8] Musalek, M. (2017). A wearable fall detector for elderly people, Proceedings of the $28^{\text {th }}$ DAAAM International Symposium, Zadar, Croatia, ISBN: 978-3-902734-11-2, ISSN 1726-9679, B. Katalinic (Ed.), pp. 1015-1020, DAAAM International, Vienna, Austria, DOI: 10.2507/28th.daaam.proceedings. 141

[9] Prausnitz, M. R. (2004). Microneedles for transdermal drug delivery, Advanced Drug Delivery Reviews, Vol. 56, No. 5, pp. 581-587, ISSN: 0169-409X

[10] Momčilović, M. \& Zygmunt, D. (2017). Design of new energy harvesting concepts based on self-winding watch mechanisms, Seminar report, Faculty of Engineering, University of Rijeka, Rijeka, Croatia, unpublished 